\title{
General iterative scheme based on the regularization for solving a constrained convex minimization problem
}

\section{Ming $\operatorname{Tian}^{*}$}

\section{"Correspondence: \\ tianming1963@126.com \\ College of Science, Civil Aviation \\ University of China, Tianjin, 300300, \\ P.R. China}

\begin{abstract}
It is well known that the regularization method plays an important role in solving a constrained convex minimization problem. In this article, we introduce implicit and explicit iterative schemes based on the regularization for solving a constrained convex minimization problem. We establish results on the strong convergence of the sequences generated by the proposed schemes to a solution of the minimization problem. Such a point is also a solution of a variational inequality. We also apply the algorithm to solve a split feasibility problem.
\end{abstract}

MSC: 47H09; 47H05; 47H06; 47J25; 47J05

Keywords: averaged mapping; gradient-projection algorithm; constrained convex minimization; regularization; split feasibility problem; variational inequality

\section{Introduction}

The gradient-projection algorithm is a classical power method for solving constrained convex optimization problems and has been studied by many authors (see [1-14] and the references therein). The method has recently been applied to solve split feasibility problems which find applications in image reconstruction and the intensity modulated radiation therapy (see [15-22]).

Consider the problem of minimizing $f$ over the constraint set $C$ (assuming that $C$ is a nonempty closed and convex subset of a real Hilbert space $H$ ). If $f: H \rightarrow \mathbb{R}$ is a convex and continuously Fréchet differentiable functional, the gradient-projection algorithm generates a sequence $\left\{x_{n}\right\}_{n=0}^{\infty}$ determined by the gradient of $f$ and the metric projection onto $C$. Under the condition that $f$ has a Lipschitz continuous and strongly monotone gradient, the sequence $\left\{x_{n}\right\}_{n=0}^{\infty}$ can be strongly convergent to a minimizer of $f$ in $C$. If the gradient of $f$ is only assumed to be inverse strongly monotone, then $\left\{x_{n}\right\}_{n=0}^{\infty}$ can only be weakly convergent if $H$ is infinite-dimensional.

Recently, $\mathrm{Xu}$ [23] gave an operator-oriented approach as an alternative to the gradientprojection method and to the relaxed gradient-projection algorithm, namely, an averaged mapping approach. He also presented two modifications of gradient-projection algorithms which are shown to have strong convergence.

On the other hand, regularization, in particular the traditional Tikhonov regularization, is usually used to solve ill-posed optimization problems [24,25]. Under some conditions, we know that the regularization method is weakly convergent.

@2013 Tian; licensee Springer. This is an Open Access article distributed under the terms of the Creative Commons Attribution License (http://creativecommons.org/licenses/by/2.0), which permits unrestricted use, distribution, and reproduction in any medium, provided the original work is properly cited. 
The purpose of this paper is to present the general iterative method combining the regularization method and the averaged mapping approach. We first propose implicit and explicit iterative schemes for solving a constrained convex minimization problem and prove that the methods converge strongly to a solution of the minimization problem, which is also a solution of the variational inequality. Furthermore, we use the above method to solve a split feasibility problem.

\section{Preliminaries}

Throughout the paper, we assume that $H$ is a real Hilbert space whose inner product and norm are denoted by $\langle\cdot, \cdot\rangle$ and $\|\cdot\|$, respectively, and that $C$ is a nonempty closed convex subset of $H$. The set of fixed points of a mapping $T$ is denoted by $\operatorname{Fix}(T)$, that is, $\operatorname{Fix}(T)=$ $\{x \in H: T x=x\}$. We write $x_{n} \rightarrow x$ to indicate that the sequence $\left\{x_{n}\right\}$ converges weakly to $x$. The fact that the sequence $\left\{x_{n}\right\}$ converges strongly to $x$ is denoted by $x_{n} \rightarrow x$. The following definition and results are needed in the subsequent sections.

Recall that a mapping $T: H \rightarrow H$ is said to be $L$-Lipschitzian if

$$
\|T x-T y\| \leq L\|x-y\|, \quad \forall x, y \in H,
$$

where $L>0$ is a constant. In particular, if $L \in[0,1)$, then $T$ is called a contraction on $H$; if $L=1$, then $T$ is called a nonexpansive mapping on $H$. T is called firmly nonexpansive if $2 T-I$ is nonexpansive, or equivalently, $\langle x-y, T x-T y\rangle \geq\|T x-T y\|^{2}, \forall x, y \in H$. Alternatively, $T$ is firmly nonexpansive if and only if $T$ can be expressed as $T=\frac{1}{2}(I+W)$, where $W: H \rightarrow H$ is nonexpansive.

Definition 2.1 A mapping $T: H \rightarrow H$ is said to be an averaged mapping if it can be written as the average of the identity $I$ and a nonexpansive mapping; that is,

$$
T=(1-\alpha) I+\alpha W,
$$

where $\alpha$ is a number in $(0,1)$ and $W: H \rightarrow H$ is nonexpansive. More precisely, when (2) holds, we say that $T$ is $\alpha$-averaged. Clearly, a firmly nonexpansive mapping (in particular, projection) is a $\frac{1}{2}$-averaged map.

Proposition 2.1 $[16,26]$ For given operators $W, T, V: H \rightarrow H$ :

(i) If $T=(1-\alpha) W+\alpha V$ for some $\alpha \in(0,1)$ and if $W$ is averaged and $V$ is nonexpansive, then $T$ is averaged.

(ii) $T$ is firmly nonexpansive if and only if the complement $I-T$ is firmly nonexpansive.

(iii) If $T=(1-\alpha) W+\alpha V$ for some $\alpha \in(0,1)$ and if $W$ is firmly nonexpansive and $V$ is nonexpansive, then $T$ is averaged.

(iv) The composite of finitely many averaged mappings is averaged. That is, if each of the mappings $\left\{T_{i}\right\}_{i=1}^{N}$ is averaged, then so is the composite $T_{1} \cdots T_{N}$. In particular, if $T_{1}$ is $\alpha_{1}$-averaged and $T_{2}$ is $\alpha_{2}$-averaged, then the composite $T_{1} T_{2}$ is $\alpha$-averaged, where $\alpha=\alpha_{1}+\alpha_{2}-\alpha_{1} \alpha_{2}$.

Recall that the metric (or nearest point) projection from $H$ onto $C$ is the mapping $P_{C}$ : $H \rightarrow C$ which assigns to each point $x \in H$ the unique point $P_{C} x \in C$ satisfying the property

$$
\left\|x-P_{C} x\right\|=\inf _{y \in C}\|x-y\|=: d(x, C)
$$


Lemma 2.1 For given $x \in H$ :

(i) $z=P_{C} x$ if and only if

$$
\langle x-z, y-z\rangle \leq 0, \quad \forall y \in C
$$

(ii) $z=P_{C} x$ if and only if

$$
\|x-z\|^{2} \leq\|x-y\|^{2}-\|y-z\|^{2}, \quad \forall y \in C
$$

(iii)

$$
\left\langle P_{C} x-P_{C} y, x-y\right\rangle \geq\left\|P_{C} x-P_{C} y\right\|^{2}, \quad \forall x, y \in H .
$$

Consequently, $P_{C}$ is nonexpansive.

Lemma 2.2 The following inequality holds in a Hilbert space X:

$$
\|x+y\|^{2} \leq\|x\|^{2}+2\langle y, x+y\rangle, \quad \forall x, y \in X .
$$

Lemma 2.3 [27] In a Hilbert space $H$, we have

$$
\|\lambda x+(1-\lambda) y\|^{2}=\lambda\|x\|^{2}+(1-\lambda)\|y\|^{2}-\lambda(1-\lambda)\|x-y\|^{2}, \quad \forall x, y \in H \text { and } \lambda \in[0,1] .
$$

Lemma 2.4 (Demiclosedness principle [27]) Let $C$ be a closed and convex subset of a Hilbert space $H$, and let $T: C \rightarrow C$ be a nonexpansive mapping with $\operatorname{Fix}(T) \neq \emptyset$. If $\left\{x_{n}\right\}_{n=1}^{\infty}$ is a sequence in $C$ weakly converging to $x$ and if $\left\{(I-T) x_{n}\right\}_{n=1}^{\infty}$ converges strongly to $y$, then $(I-T) x=y$. In particular, if $y=0$, then $x \in \operatorname{Fix}(T)$.

Definition 2.2 A nonlinear operator $G$ with domain $D(G) \subseteq H$ and range $R(G) \subseteq H$ is said to be:

(i) monotone if

$$
\langle x-y, G x-G y\rangle \geq 0, \quad \forall x, y \in D(G)
$$

(ii) $\beta$-strongly monotone if there exists $\beta>0$ such that

$$
\langle x-y, G x-G y\rangle \geq \beta\|x-y\|^{2}, \quad \forall x, y \in D(G),
$$

(iii) $v$-inverse strongly monotone (for short, $v$-ism) if there exists $v>0$ such that

$$
\langle x-y, G x-G y\rangle \geq v\|G x-G y\|^{2}, \quad \forall x, y \in D(G) .
$$

Proposition 2.2 [16] Let $T: H \rightarrow H$ be an operator from $H$ to itself.

(i) $T$ is nonexpansive if and only if the complement $I-T$ is $\frac{1}{2}-$ ism.

(ii) If $T$ is $v$-ism, then for $\gamma>0, \gamma T$ is $\frac{v}{\gamma}$-ism.

(iii) $T$ is averaged if and only if the complement $I-T$ is $v$-ism for some $v>1 / 2$. Indeed, for $\alpha \in(0,1), T$ is $\alpha$-averaged if and only if $I-T$ is $\frac{1}{2 \alpha}$-ism. 
Lemma 2.5 [6] Assume that $\left\{a_{n}\right\}$ is a sequence of nonnegative real numbers such that

$$
a_{n+1} \leq\left(1-\gamma_{n}\right) a_{n}+\gamma_{n} \delta_{n}, \quad n \geq 0,
$$

where $\left\{\gamma_{n}\right\}$ is a sequence in $(0,1)$ and $\left\{\delta_{n}\right\}$ is a sequence in $\mathbb{R}$ such that

(i) $\sum_{n=1}^{\infty} \gamma_{n}=\infty$;

(ii) $\limsup _{n \rightarrow \infty} \delta_{n} \leq 0$ or $\sum_{n=1}^{\infty} \gamma_{n}\left|\delta_{n}\right|<\infty$.

Then $\lim _{n \rightarrow \infty} a_{n}=0$.

\section{Main results}

We now look at the constrained convex minimization problem:

$$
\min _{x \in C} f(x)
$$

where $C$ is a closed and convex subset of a Hilbert space $H$ and $f: C \rightarrow \mathbb{R}$ is a real-valued convex function. Assume that problem (4) is consistent, let $S$ denote the solution set. If $f$ is Fréchet differentiable, then the gradient-projection algorithm (GPA) generates a sequence $\left\{x_{n}\right\}_{n=0}^{\infty}$ according to the recursive formula

$$
x_{n+1}=\operatorname{Proj}_{C}(I-\gamma \nabla f)\left(x_{n}\right), \quad n \geq 0,
$$

or more generally,

$$
x_{n+1}=\operatorname{Proj}_{C}\left(I-\gamma_{n} \nabla f\right)\left(x_{n}\right), \quad n \geq 0,
$$

where, in both (5) and (6), the initial guess $x_{0}$ is taken from $C$ arbitrarily, the parameters $\gamma$ or $\gamma_{n}$ are positive real numbers.

As a matter of fact, it is known that if $\nabla f$ fails to be strongly monotone, and is only $\frac{1}{L}$-ism, namely, there is a constant $L>0$ such that

$$
\langle x-y, \nabla f(x)-\nabla f(y)\rangle \geq \frac{1}{L}\|\nabla f(x)-\nabla f(y)\|^{2}, \quad x, y \in C,
$$

under some assumption for $\gamma$ or $\gamma_{n}$, then algorithms (5) and (6) can still converge in the weak topology.

Now consider the regularized minimization problem

$$
\min _{x \in C} f_{\alpha}(x):=\min _{x \in C}\left\{f(x)+\frac{\alpha}{2}\|x\|^{2}\right\}
$$

where $\alpha>0$ is the regularization parameter, and again $f$ is convex with a $\frac{1}{L}$-ism gradient $\nabla f$.

It is known that the regularization method is defined as follows:

$$
x_{n+1}=\operatorname{Proj}_{C}\left(I-\gamma \nabla f_{\alpha_{n}}\right)\left(x_{n}\right) .
$$

We also know that $\left\{x_{n}\right\} \rightarrow \tilde{x}$, where $\tilde{x}$ is a solution of constrained convex minimization problem (4). 
Let $h: C \rightarrow H$ be a contraction with a constant $\rho>0$. In this section, we introduce the following implicit scheme generating a net $\left\{x_{s, t_{s}}\right\}$ in an implicit way:

$$
x_{s, t_{s}}=P_{C}\left[\operatorname{sh}\left(x_{s, t_{s}}\right)+(1-s) T_{t_{s}} x_{s, t_{s}}\right]
$$

where $0<\gamma<\frac{2}{L}, t_{s} \in\left(0, \frac{2}{\gamma}-L\right)$. Let $T_{t_{s}}$ and $s$ satisfy the following conditions:

(i) $\lambda:=\lambda\left(t_{s}\right)=\frac{2-\gamma\left(L+t_{s}\right)}{4}$;

(ii) $P_{C}\left(I-\gamma \nabla f_{t_{s}}\right)=\lambda I+(1-\lambda) T_{t_{s}}$.

Consider a mapping

$$
Q_{s} x=P_{C}\left[\operatorname{sh}(x)+(1-s) T_{t_{s}} x\right], \quad \forall x \in C .
$$

It is easy to see that $Q_{s}$ is a contraction. Indeed, we have

$$
\begin{aligned}
\left\|Q_{s} x-Q_{s} y\right\| & \leq\left\|s h(x)+(1-s) T_{t_{s}} x-\left[\operatorname{sh}(y)+(1-s) T_{t_{s}} y\right]\right\| \\
& \leq \rho s\|x-y\|+(1-s)\|x-y\|=[1-(1-\rho) s]\|x-y\| .
\end{aligned}
$$

Hence, $Q_{s}$ has a unique fixed point in $C$, denoted by $x_{s, t_{s}}$, which uniquely solves fixed point equation (7).

We proved the strong convergence of $\left\{x_{s, t_{s}}\right\}_{t_{s} \in\left(0, \frac{2}{\gamma}-L\right)}$ to a solution $x^{*}$ of the minimization problem

$$
\left\langle(I-h) x^{*}, x^{*}-z\right\rangle \leq 0, \quad \forall z \in S
$$

For a given arbitrary guess $x_{0} \in C$ and a sequence $\left\{\alpha_{n}\right\} \in\left(0, \frac{2}{\gamma}-L\right)$, we also propose the following explicit scheme that generates a sequence $\left\{x_{n}\right\}$ in an explicit way:

$$
x_{n+1}=P_{C}\left[\theta_{n} h\left(x_{n}\right)+\left(1-\theta_{n}\right) T_{n} x_{n}\right], \quad n \geq 0,
$$

where $0<\gamma<\frac{2}{L}, \lambda_{n}=\frac{2-\gamma\left(L+\alpha_{n}\right)}{4}$ and $P_{C}\left(I-\gamma \nabla f_{\alpha_{n}}\right)=\lambda_{n} I+\left(1-\lambda_{n}\right) T_{n}$ for each $n \geq 0$. It is proven that this sequence $\left\{x_{n}\right\}$ converges strongly to a minimizer $x^{*} \in S$ of (4).

\subsection{Convergence of the implicit scheme}

Proposition 3.1 If $0<\gamma<\frac{2}{L}, \alpha \in\left(0, \frac{2}{\gamma}-L\right), \nabla f$ is $\frac{1}{L}$-ism, then

$$
\begin{aligned}
& \operatorname{Proj}_{C}\left(I-\gamma \nabla f_{\alpha}\right)=\left(1-\mu_{\alpha}\right) I+\mu_{\alpha} T_{\alpha}, \\
& \operatorname{Proj}_{C}(I-\gamma \nabla f)=(1-\mu) I+\mu T,
\end{aligned}
$$

where $\mu_{\alpha}=\frac{2+\gamma(L+\alpha)}{4}, \mu=\frac{2+\gamma L}{4}$.

In addition, for $\forall x \in C$,

$$
\left\|T_{\alpha} x-T x\right\| \leq \alpha M(x)
$$

where

$$
M(x)=\gamma(5\|x\|+\|T x\|) .
$$


Proof Since $\nabla f$ is $\frac{1}{L}$-ism, so $\gamma \nabla f_{\alpha}$ is $\frac{1}{\gamma(L+\alpha)}$-ism, by Proposition 2.2, $I-\gamma \nabla f_{\alpha}$ is $\frac{\gamma(L+\alpha)}{2}$ averaged, because $\operatorname{Proj}_{C}$ is $\frac{1}{2}$-averaged, by Proposition 2.1, $\operatorname{Proj}_{C}\left(I-\gamma \nabla f_{\alpha}\right)$ is $\mu_{\alpha}$-averaged, i.e.,

$\operatorname{Proj}_{C}\left(I-\gamma \nabla f_{\alpha}\right)=\left(1-\mu_{\alpha}\right) I+\mu_{\alpha} T_{\alpha}$,

where $\mu_{\alpha}=\frac{2+\gamma(L+\alpha)}{4}$. The same case holds for $\operatorname{Proj}_{C}(I-\gamma \nabla f)$.

Hence,

$$
\begin{aligned}
& \left\|\operatorname{Proj}_{C}\left(I-\gamma \nabla f_{\alpha}\right) x-\operatorname{Proj}_{C}\left(I-\gamma \nabla f_{\alpha}\right) x\right\| \\
& \quad=\left\|\left(\mu-\mu_{\alpha}\right) x+\mu_{\alpha} T_{\alpha} x-\mu T x\right\| \\
& \quad \leq\left\|\left(I-\gamma \nabla f_{\alpha}\right) x-(I-\gamma \nabla f) x\right\| \\
& \quad=\gamma\left\|\nabla f_{\alpha}(x)-\nabla f(x)\right\|=\alpha \gamma\|x\|,
\end{aligned}
$$

then

$$
\begin{aligned}
& \left\|\mu_{\alpha}\left(T_{\alpha} x\right)-\mu T x\right\| \leq\left|\mu-\mu_{\alpha}\right|\|x\|+\alpha \gamma\|x\|, \\
& \left\|T_{\alpha} x-T x\right\| \leq \frac{\alpha \gamma(5\|x\|+\|T x\|)}{2+\gamma(L+\alpha)} \leq \alpha M(x),
\end{aligned}
$$

where $M(x)=\gamma(5\|x\|+\|T x\|)$.

Proposition 3.2 Let $h: C \rightarrow H$ be a contraction with $0<\rho<1$ and $0<\gamma<\frac{2}{L}$, let $t_{s}$ be continuous with respect to $s, t_{s}=o(s)$. Suppose that problem (4) is consistent, let $S$ denote the solution set for each $s \in(0,1)$, and let $x_{s, t_{s}}$ denote a unique solution of fixed point equation (7). Then the following properties hold for the net $\left\{x_{s, t_{s}}\right\}$ :

(i) $\left\{x_{s, t_{s}}\right\}_{t_{s} \in\left(0, \frac{2}{\gamma}-L\right)}$ is bounded;

(ii) $\lim _{s \rightarrow 0}\left\|x_{s, t_{s}}-T_{t_{s}} x_{s, t_{s}}\right\|=0$;

(iii) $x_{s, t_{s}}$ defines a continuous curve from $(0,1)$ into $C$.

Proof (i) Take any $p \in S$, then

$$
x_{s, t_{s}}-p=P_{C}\left[s h\left(x_{s, t_{s}}\right)+(1-s) T_{t_{s}} x_{s, t_{s}}\right]-P_{C} p .
$$

Therefore,

$$
\begin{aligned}
\left\|x_{s, t_{s}}-p\right\| & \leq s \rho\left\|x_{s, t_{s}}-p\right\|+s\|h(p)-p\|+(1-s)\left\|T_{t_{s}} x_{s, t_{s}}-T p\right\| \\
& \leq s \rho\left\|x_{s, t_{s}}-p\right\|+s\|h(p)-p\|+(1-s)\left[\left\|T_{t_{s}} x_{s, t_{s}}-T_{t_{s}} p\right\|+\left\|T_{t_{s}} p-T p\right\|\right] \\
& \leq[1-(1-\rho) s]\left\|x_{s, t_{s}}-p\right\|+s\|h(p)-p\|+(1-s) t_{s}\|M(p)\|
\end{aligned}
$$

hence,

$$
\left\|x_{s, t_{s}}-p\right\| \leq \frac{\|h(p)-p\|}{1-\rho}+(1-s) \frac{t_{s}}{s(1-\rho)}\|M(p)\| .
$$

So, $\left\{x_{s, t_{s}}\right\}$ is bounded. 
(ii)

$$
\left\|x_{s, t_{s}}-T_{t_{s}} x_{s, t_{s}}\right\| \leq\left\|s h\left(x_{s, t_{s}}\right)-s T_{t_{s}} x_{s, t_{s}}\right\| \rightarrow 0 .
$$

(iii) Take $s, s_{0} \in(0,1)$, and calculate

$$
\begin{aligned}
& \left\|x_{s, t_{s}}-x_{s_{0}, t_{s_{0}}}\right\| \leq\left\|\operatorname{sh}\left(x_{s, t_{s}}\right)+(1-s) T t_{s} x_{s, t_{s}}-\left[s_{0} h\left(x_{s_{0}, t_{s_{0}}}\right)+\left(1-s_{0}\right) T_{t_{s_{0}}} x_{s_{0}, t_{s_{0}}}\right]\right\| \\
& =\| s\left(h\left(x_{s, t_{s}}\right)-h\left(x_{s_{0}, t_{s_{0}}}\right)\right)+\left(s-s_{0}\right)\left[h\left(x_{s_{0}, t_{s_{0}}}\right)-T_{t_{s_{0}}} x_{s_{0}, t_{s_{0}}}\right] \\
& +(1-s)\left[T_{t_{s}} x_{s, t_{s}}-T_{t_{s}} x_{s_{0}, t_{t_{0}}}\right]+(1-s)\left[T_{t_{s}} x_{s_{0}, t_{s_{0}}}-T_{t_{s_{0}}} x_{s_{0}, t_{s_{0}}}\right] \| \\
& \leq s \rho\left\|x_{s, t_{s}}-x_{s_{0}, t_{s_{0}}}\right\|+(1-s)\left\|x_{s, t_{s}}-x_{s_{0}, t_{s_{0}}}\right\| \\
& +(1-s)\left\|T_{t_{s}} x_{s_{0}, t_{s_{0}}}-T_{t_{s_{0}}} x_{s_{0}, t_{s_{0}}}\right\|+\left|s-s_{0}\right|\left\|h\left(x_{s_{0}, t_{s_{0}}}\right)-T_{t_{s_{0}}} x_{s_{0}, t_{s_{0}}}\right\|, \\
& \left\|T_{t_{s}} x_{s_{0}, t_{s_{0}}}-T_{t_{s_{0}}} x_{s_{0}, t_{s_{0}}}\right\| \\
& =\| \frac{4 P_{C}\left(I-\gamma \nabla f_{t_{s}}\right)-\left[2-\gamma\left(L+t_{s}\right)\right] I}{2+\gamma\left(L+t_{s}\right)} x_{s_{0}, t_{s_{0}}} \\
& -\frac{4 P_{C}\left(I-\gamma \nabla f_{t_{0}}\right)-\left[2-\gamma\left(L+t_{s_{0}}\right)\right] I}{2+\gamma\left(L+t_{s_{0}}\right)} x_{s_{0}, t_{s_{0}}} \\
& \leq\left\|\frac{4 P_{C}\left(I-\gamma \nabla f_{t_{s}}\right)}{2+\gamma\left(L+t_{s}\right)} x_{s_{0}, t_{s_{0}}}-\frac{4 P_{C}\left(I-\gamma \nabla f_{t_{s_{0}}}\right)}{2+\gamma\left(L+t_{s_{0}}\right)} x_{s_{0}, t_{s_{0}}}\right\| \\
& +\left\|\frac{-\left[2-\gamma\left(L+t_{s}\right)\right]}{2+\gamma\left(L+t_{s}\right)} x_{s_{0}, t_{s_{0}}}+\frac{\left[2-\gamma\left(L+t_{s_{0}}\right)\right]}{2+\gamma\left(L+t_{s_{0}}\right)} x_{s_{0}, t_{s_{0}}}\right\| \\
& =\left\|\frac{4\left[2+\gamma\left(L+t_{s_{0}}\right)\right] P_{C}\left(I-\gamma \nabla f_{t_{s}}\right) x_{s_{0}, t_{s_{0}}}-4\left[2+\gamma\left(L+t_{s}\right)\right] P_{C}\left(I-\gamma \nabla f_{t_{s_{0}}}\right) x_{s_{0}, t_{s_{0}}}}{\left[2+\gamma\left(L+t_{s}\right)\right]\left[2+\gamma\left(L+t_{s_{0}}\right)\right]}\right\| \\
& +\frac{4 \gamma\left|t_{s}-t_{s_{0}}\right|\left\|x_{s_{0}, t_{s_{0}}}\right\|}{\left[2+\gamma\left(L+t_{s}\right)\right]\left[2+\gamma\left(L+t_{s_{0}}\right)\right]} \\
& =\| \frac{4 \gamma\left(t_{s_{0}}-t_{s}\right) P_{C}\left(I-\gamma \nabla f_{t_{s}}\right) x_{s_{0}, t_{s_{0}}}}{\left[2+\gamma\left(L+t_{s}\right)\right]\left[2+\gamma\left(L+t_{s_{0}}\right)\right]} \\
& +\frac{4\left(2+\gamma\left(L+t_{s}\right)\right)\left[P_{C}\left(I-\gamma \nabla f_{t_{s}}\right) x_{s_{0}, t_{s_{0}}}-P_{C}\left(I-\gamma \nabla f_{t_{s_{0}}}\right) x_{s_{0}, t_{s_{0}}}\right]}{\left[2+\gamma\left(L+t_{s}\right)\right]\left[2+\gamma\left(L+t_{s_{0}}\right)\right]} \| \\
& +\frac{4 \gamma\left|t_{s}-t_{s_{0}}\right|\left\|x_{s_{0}, t_{s_{0}}}\right\|}{\left[2+\gamma\left(L+t_{s}\right)\right]\left[2+\gamma\left(L+t_{s_{0}}\right)\right]} \\
& \leq M_{1}\left|t_{s}-t_{s_{0}}\right| .
\end{aligned}
$$

So, by (12) and (13),

$$
\left\|x_{s, t_{s}}-x_{s_{0}, t_{s_{0}}}\right\| \rightarrow 0 \quad\left(s \rightarrow s_{0}\right)
$$

Theorem 3.1 Assume that minimization problem (4) is consistent, and let $S$ denote the solution set. Assume that the gradient $\nabla f$ is $\frac{1}{L}$-ism. Let $h: C \rightarrow H$ be a $\rho$-contraction with $\rho \in[0,1)$,

$$
x_{s, t_{s}}=P_{C}\left[\operatorname{sh}\left(x_{s, t_{s}}\right)+(1-s) T_{t_{s}} x_{s, t_{s}}\right] \text {, }
$$


where $0<\gamma<\frac{2}{L}, t_{s} \in\left(0, \frac{2}{\gamma}-L\right), t_{s}=o(s)$. Let $T_{t_{s}}$ satisfy the following conditions:

(i) $\lambda:=\lambda\left(t_{s}\right)=\frac{2-\gamma\left(L+t_{s}\right)}{4}$;

(ii) $P_{C}\left(I-\gamma \nabla f_{t_{s}}\right)=\lambda I+(1-\lambda) T_{t_{s}}$

Then the net $\left\{x_{s, t_{s}}\right\}$ converges strongly as $s \rightarrow 0$ to a minimizer of problem (4), which is also the unique solution of the variational inequality

$x^{*} \in S, \quad\left\langle(I-h) x^{*}, x-x^{*}\right\rangle \geq 0, \quad \forall x \in S$.

Proof Set $\operatorname{Proj}_{C}(I-\gamma \nabla f)=(1-\tau) I+\tau T, \tau=\frac{2-\gamma L}{4}$. Let $y_{s, t_{s}}=\operatorname{sh}\left(x_{s, t_{s}}\right)+(1-s) T_{t_{s}} x_{s, t_{s}}, t_{s} \in$ $\left(0, \frac{2}{\gamma}-L\right)$.

We then have $x_{s, t_{s}}=P_{C} y_{s, t_{S}}$. For any given $z \in S, z=P_{C}(I-\gamma \nabla f) z$, we obtain

$$
\begin{aligned}
x_{s, t_{s}}-z & =P_{C} y_{s, t_{s}}-y_{s, t_{s}}+y_{s, t_{s}}-z \\
& =P_{C} y_{s, t_{s}}-y_{s, t_{s}}+s h\left(x_{s, t_{s}}\right)+(1-s) T_{t_{s}} x_{s, t_{s}}-T z \\
& =P_{C} y_{s, t_{s}}-y_{s, t_{s}}+s\left[h\left(x_{s, t_{s}}\right)-h(z)\right]+s[h(z)-T z]+(1-s)\left[T_{t_{s}} x_{s, t_{s}}-T z\right] .
\end{aligned}
$$

Next we prove that $\left\{x_{s, t_{s}}\right\} \rightarrow x^{*} \in S$, which is also the unique solution of the variational inequality. We have

$$
\begin{aligned}
\left\|x_{s, t_{s}}-z\right\|^{2}= & \left\langle P_{C} y_{s, t_{s}}-y_{s, t_{s}}, P_{C} y_{s, t_{s}}-z\right\rangle+s\left\langle h\left(x_{s, t_{s}}\right)-h(z), x_{s, t_{s}}-z\right\rangle \\
& +s\left\langle h(z)-T z, x_{s, t_{s}}-z\right\rangle+(1-s)\left\langle T_{t_{s}} x_{s, t_{s}}-T z, x_{s, t_{s}}-z\right\rangle \\
\leq & s \rho\left\|x_{s, t_{s}}-z\right\|^{2}+s\left\langle h(z)-T z, x_{s, t_{s}}-z\right\rangle+(1-s)\left\langle T_{t_{s}} x_{s, t_{s}}-T z, x_{s, t_{s}}-z\right\rangle \\
\leq & {[1-(1-\rho) s]\left\|x_{s, t_{s}}-z\right\|^{2}+s\left\langle h(z)-T z, x_{s, t_{s}}-z\right\rangle } \\
& +(1-s) t_{s}\|M(z)\|\left\|x_{s, t_{s}}-z\right\| .
\end{aligned}
$$

So,

$$
\left\|x_{s, t_{s}}-z\right\|^{2} \leq \frac{\left\langle h(z)-T z, x_{s, t_{s}}-z\right\rangle}{1-\rho}+\frac{(1-s) t_{s}\|M(z)\|\left\|x_{s, t_{s}}-z\right\|}{s(1-\rho)} .
$$

Then if $x_{s_{n}, t_{s_{n}}} \rightarrow p$, then $x_{s_{n}, t_{s_{n}}} \rightarrow p$. Next, we prove that

$$
\left\|x_{s, t_{s}}-\operatorname{Proj}_{C}(I-\gamma \nabla f) x_{s, t_{s}}\right\| \rightarrow 0 \text {. }
$$

Since

$$
\begin{aligned}
\left\|\operatorname{Proj}_{C}\left(I-\gamma \nabla f_{t_{s}}\right) x_{s, t_{s}}-x_{s, t_{s}}\right\| & =\left\|\lambda x_{s, t_{s}}+(1-\lambda) T_{t_{s}}\left(x_{s, t_{s}}\right)-x_{s, t_{s}}\right\| \\
& \leq\left\|T_{t_{s}}\left(x_{s, t_{s}}\right)-x_{s, t_{s}}\right\| .
\end{aligned}
$$

So,

$$
\left\|x_{s, t_{s}}-\operatorname{Proj}_{C}(I-\gamma \nabla f) x_{s, t_{s}}\right\| \leq \gamma t_{s}\left\|x_{s, t_{s}}\right\|+\left\|T_{t_{s}}\left(x_{s, t_{s}}\right)-x_{s, t_{s}}\right\| \rightarrow 0 .
$$

Finally, we prove that $\left\{x_{s, t_{s}}\right\} \rightarrow x^{*} \in S$, which is also the unique solution of the variational inequality. We only need to prove that if $x_{s_{n}, t_{s_{n}}} \rightarrow \tilde{x}$, then

$$
\langle(I-h) \tilde{x}, x-\tilde{x}\rangle \geq 0, \quad \forall x \in S .
$$


Suppose that $x_{s_{n}, t_{s n}}-\tilde{x}$, by Lemma 2.4 and $\left\|x_{s, t_{s}}-\operatorname{Proj}_{C}(I-\gamma \nabla f) x_{s, t_{s}}\right\| \rightarrow 0, \tilde{x}=\operatorname{Proj}_{C}(I-$ $\gamma \nabla f) \tilde{x}$, it follows that $\tilde{x} \in S$. Note that $x_{s_{n}, t_{s_{n}}} \rightarrow \tilde{x}$ by (14). From the definition

$$
x_{s, t_{s}}=P_{C}\left[\operatorname{sh}\left(x_{s, t_{s}}\right)+(1-s) T_{t_{s}} x_{s, t_{s}}\right]
$$

we have

$$
(I-h)\left(x_{s_{n}, t_{s_{n}}}\right)=\frac{1}{s_{n}}\left(P_{C} y_{s_{n}, t_{s_{n}}}-y_{s_{n}, t_{s_{n}}}\right)-\frac{1}{s_{n}}\left[\left(I-T_{t_{s_{n}}}\right) x_{s_{n}, t_{s_{n}}}\right]+\left[x_{s_{n}, t_{s_{n}}}-T_{t_{s_{n}}} x_{s_{n}, t_{s_{n}}}\right] .
$$

So

$$
\begin{aligned}
\langle(I- & \left.h)\left(x_{s_{n}, t_{s_{n}}}\right), x_{s_{n}, t_{s_{n}}}-z\right\rangle \\
= & \frac{1}{s_{n}}\left\langle P_{C} y_{s_{n}, t_{s_{n}}}-y_{s_{n}, t_{s_{n}}}, x_{s_{n}, t_{s_{n}}}-z\right\rangle \\
& -\frac{1}{s_{n}}\left\langle x_{s_{n}, t_{s_{n}}}-T_{t_{s_{n}}} x_{s_{n}, t_{s_{n}}}, x_{s_{n}, t_{s_{n}}}-z\right\rangle+\left\langle x_{s_{n}, t_{s_{n}}}-T_{t_{s_{n}}} x_{s_{n}, t_{s_{n}}}, x_{s_{n}, t_{s_{n}}}-z\right\rangle \\
\leq & -\frac{1}{s_{n}}\left\langle\left(I-T_{t_{s_{n}}}\right) x_{s_{n}, t_{s_{n}}}, x_{s_{n}, t_{s_{n}}}-z\right\rangle+\left\langle\left(I-T_{t_{s_{n}}}\right) x_{s_{n}, t_{s_{n}}}, x_{s_{n}, t_{s_{n}}}-z\right\rangle \\
\leq & -\frac{1}{s_{n}}\left[\left\langle(I-T) x_{s_{n}, t_{s_{n}}}, x_{s_{n}, t_{s_{n}}}-z\right\rangle-\frac{1}{s_{n}}\left\langle\left(T-T_{t_{s_{n}}}\right) x_{s_{n}, t_{s_{n}}}, x_{s_{n}, t_{s_{n}}}-z\right\rangle\right] \\
& +\left\langle x_{s_{n}, t_{s_{n}}}-T_{t_{s_{n}}} x_{s_{n}, t_{s_{n}}}, x_{s_{n}, t_{s_{n}}}-z\right\rangle,
\end{aligned}
$$

then

$$
\langle(I-h) \tilde{x}, \tilde{x}-z\rangle=\lim _{n \rightarrow \infty}\left\langle(I-h)\left(x_{s_{n}, t_{s_{n}}}\right), x_{s_{n}, t_{s_{n}}}-z\right\rangle \leq 0 .
$$

So, $\left\{x_{s, t_{s}}\right\} \rightarrow x^{*} \in S$, which is also the unique solution of the variational inequality.

\subsection{Convergence of the explicit scheme}

Theorem 3.2 Assume that minimization problem (4) is consistent, and let $S$ denote the solution set. Assume that the gradient $\nabla f$ is $\frac{1}{L}$-ism. Let $h: C \rightarrow H$ be a $\rho$-contraction with $\rho \in[0,1)$. Let a sequence $\left\{x_{n}\right\}_{n=0}^{\infty}$ be generated by the following hybrid gradient projection algorithm:

$$
x_{n+1}=P_{c}\left[\theta_{n} h\left(x_{n}\right)+\left(1-\theta_{n}\right) T_{n}\left(x_{n}\right)\right], \quad n=0,1,2, \ldots,
$$

where $0<\gamma<\frac{2}{L}, P_{c}\left[I-\gamma \nabla f_{\alpha_{n}}\right]=\lambda_{n} I+\left(1-\lambda_{n}\right) T_{n}$ and $\lambda_{n}=\frac{2-\gamma\left(L+\alpha_{n}\right)}{4}$, and, in addition, assume that the following conditions are satisfied for $\left\{\theta_{n}\right\}_{n=0}^{\infty}$ and $\left\{\alpha_{n}\right\}_{n=0}^{\infty}$ :

(i) $\theta_{n} \rightarrow 0 ; \alpha_{n}=o\left(\theta_{n}\right)$;

(ii) $\sum_{n=0}^{\infty} \theta_{n}=\infty$;

(iii) $\sum_{n=0}^{\infty}\left|\theta_{n+1}-\theta_{n}\right|<\infty$;

(iv) $\sum_{n=0}^{\infty}\left|\alpha_{n+1}-\alpha_{n}\right|<\infty$.

Then the sequence $\left\{x_{n}\right\}_{n=0}^{\infty}$ converges in norm to a minimizer of (4) which is also the unique solution of the variational inequality $(V I)$

$$
x^{*} \in S, \quad\left\langle(I-h) x^{*}, x-x^{*}\right\rangle \geq 0, \quad \forall x \in S .
$$


In other words, $x^{*}$ is the unique fixed point of the contraction $\operatorname{Proj}_{S} h$,

$$
x^{*}=\operatorname{Proj}_{S} h\left(x^{*}\right) .
$$

Proof $\left(1^{\circ}\right)$ We first prove that $\left\{x_{n}\right\}_{n=0}^{\infty}$ is bounded. Set $\operatorname{Proj}_{C}(I-\gamma \nabla f)=(1-\tau) I+\tau T, \tau=$ $\frac{2-\gamma L}{4}$. Indeed, we have, for $\tilde{x} \in S$,

$$
\begin{aligned}
& \left\|x_{n+1}-\tilde{x}\right\| \\
& \quad=\left\|P_{C}\left[\theta_{n} h\left(x_{n}\right)+\left(1-\theta_{n}\right) T_{n}\left(x_{n}\right)\right]-P_{C} \tilde{x}\right\| \\
& \quad \leq\left\|\theta_{n} h\left(x_{n}\right)+\left(1-\theta_{n}\right) T_{n}\left(x_{n}\right)-\tilde{x}\right\| \\
& \quad=\left\|\theta_{n}\left(h\left(x_{n}\right)-h(\tilde{x})\right)+\theta_{n}(h(\tilde{x})-\tilde{x})+\left(1-\theta_{n}\right)\left(T_{n}\left(x_{n}\right)-\tilde{x}\right)\right\| \\
& \quad \leq \theta_{n} \rho\left\|x_{n}-\tilde{x}\right\|+\theta_{n}\|h(\tilde{x})-\tilde{x}\|+\left(1-\theta_{n}\right)\left[\left\|x_{n}-\tilde{x}\right\|+\left\|T_{n}(\tilde{x})-T(\tilde{x})\right\|\right] \\
& \quad \leq\left(1-(1-\rho) \theta_{n}\right)\left\|x_{n}-\tilde{x}\right\|+\theta_{n}\|h(\tilde{x})-\tilde{x}\|+\alpha_{n}\|M(\tilde{x})\| \\
& \quad \leq \max \left\{\left\|x_{n}-\tilde{x}\right\|, \frac{1}{1-\rho}[\|h(\tilde{x})-\tilde{x}\|+\|M(\tilde{x})\|]\right\} .
\end{aligned}
$$

So, $\left\{x_{n}\right\}$ is bounded.

$\left(2^{\circ}\right)$ Next we prove that $\left\|x_{n+1}-x_{n}\right\| \rightarrow 0$ as $n \rightarrow \infty$.

$$
\begin{aligned}
&\left\|x_{n+1}-x_{n}\right\| \\
&=\left\|P_{C}\left[\theta_{n} h\left(x_{n}\right)+\left(1-\theta_{n}\right) T_{n} x_{n}\right]-P_{C}\left[\theta_{n-1} h\left(x_{n-1}\right)+\left(1-\theta_{n-1}\right) T_{n-1} x_{n-1}\right]\right\| \\
& \leq\left\|\left[\theta_{n} h\left(x_{n}\right)+\left(1-\theta_{n}\right) T_{n} x_{n}\right]-\left[\theta_{n-1} h\left(x_{n-1}\right)+\left(1-\theta_{n-1}\right) T_{n-1} x_{n-1}\right]\right\| \\
&= \| \theta_{n}\left(h\left(x_{n}\right)-h\left(x_{n-1}\right)\right)+\left(1-\theta_{n}\right)\left(T_{n} x_{n}-T_{n} x_{n-1}\right) \\
&+\left(\theta_{n}-\theta_{n-1}\right)\left(h\left(x_{n-1}\right)-T_{n} x_{n-1}\right)+\left(1-\theta_{n-1}\right)\left(T_{n} x_{n-1}-T_{n-1} x_{n-1}\right) \| \\
& \leq {\left[1-(1-\rho) \theta_{n}\right]\left\|x_{n}-x_{n-1}\right\|+M_{2}\left|\theta_{n}-\theta_{n-1}\right|+\left(1-\theta_{n-1}\right)\left\|T_{n} x_{n-1}-T_{n-1} x_{n-1}\right\|, }
\end{aligned}
$$

but

$$
\begin{aligned}
\| & T_{n} x_{n-1}-T_{n-1} x_{n-1} \| \\
= & \| \frac{4 P_{C}\left(I-\gamma \nabla f_{\alpha_{n}}\right)-\left[2-\gamma\left(L+\alpha_{n}\right)\right] I}{2+\gamma\left(L+\alpha_{n}\right)} x_{n-1} \\
& -\frac{4 P_{C}\left(I-\gamma \nabla f_{\alpha_{n-1}}\right)-\left[2-\gamma\left(L+\alpha_{n-1}\right)\right] I}{2+\gamma\left(L+\alpha_{n-1}\right)} x_{n-1} \| \\
\leq & \left\|\frac{4 P_{C}\left(I-\gamma \nabla f_{\alpha_{n}}\right)}{2+\gamma\left(L+\alpha_{n}\right)} x_{n-1}-\frac{4 P_{C}\left(I-\gamma \nabla f_{\alpha_{n-1}}\right)}{2+\gamma\left(L+\alpha_{n-1}\right)} x_{n-1}\right\| \\
& +\left\|\frac{-\left[2-\gamma\left(L+\alpha_{n}\right)\right]}{2+\gamma\left(L+\alpha_{n}\right)} x_{n-1}+\frac{\left[2-\gamma\left(L+\alpha_{n-1}\right)\right]}{2+\gamma\left(L+\alpha_{n-1}\right)} x_{n-1}\right\| \\
= & \left\|\frac{4\left[2+\gamma\left(L+\alpha_{n-1}\right)\right] P_{C}\left(I-\gamma \nabla f_{\alpha_{n}}\right) x_{n-1}-4\left[2+\gamma\left(L+\alpha_{n}\right)\right] P_{C}\left(I-\gamma \nabla f_{\alpha_{n-1}}\right) x_{n-1}}{\left[2+\gamma\left(L+\alpha_{n}\right)\right]\left[2+\gamma\left(L+\alpha_{n-1}\right)\right]}\right\| \\
& +\frac{4 \gamma\left|\alpha_{n}-\alpha_{n-1}\right|\left\|x_{n-1}\right\|}{\left[2+\gamma\left(L+\alpha_{n}\right)\right]\left[2+\gamma\left(L+\alpha_{n-1}\right)\right]} \\
= & \| \frac{4 \gamma\left(\alpha_{n-1}-\alpha_{n}\right) P_{C}\left(I-\gamma \nabla f_{\alpha_{n}}\right) x_{n-1}}{\left[2+\gamma\left(L+\alpha_{n}\right)\right]\left[2+\gamma\left(L+\alpha_{n-1}\right)\right]}
\end{aligned}
$$




$$
\begin{aligned}
& +\frac{4\left(2+\gamma\left(L+\alpha_{n}\right)\right)\left[P_{C}\left(I-\gamma \nabla f_{\alpha_{n}}\right) x_{n-1}-P_{C}\left(I-\gamma \nabla f_{\alpha_{n-1}}\right) x_{n-1}\right] \|}{\left[2+\gamma\left(L+\alpha_{n}\right)\right]\left[2+\gamma\left(L+\alpha_{n-1}\right)\right]} \| \\
& +\frac{4 \gamma\left|\alpha_{n}-\alpha_{n-1}\right|\left\|x_{n-1}\right\|}{\left[2+\gamma\left(L+\alpha_{n}\right)\right]\left[2+\gamma\left(L+\alpha_{n-1}\right)\right]} \\
\leq & \frac{4 \gamma\left|\alpha_{n-1}-\alpha_{n}\right|\left\|P_{C}\left(I-\gamma \nabla f_{\alpha_{n}}\right) x_{n-1}\right\|}{\left[2+\gamma\left(L+\alpha_{n}\right)\right]\left[2+\gamma\left(L+\alpha_{n-1}\right)\right]} \\
& +\frac{4 \gamma\left|\alpha_{n-1}-\alpha_{n}\right|\left[2+\gamma\left(L+\alpha_{n}\right)\right]\left\|x_{n-1}\right\|}{\left[2+\gamma\left(L+\alpha_{n}\right)\right]\left[2+\gamma\left(L+\alpha_{n-1}\right)\right]} \\
& +\frac{4 \gamma\left|\alpha_{n}-\alpha_{n-1}\right|\left\|x_{n-1}\right\|}{\left[2+\gamma\left(L+\alpha_{n}\right)\right]\left[2+\gamma\left(L+\alpha_{n-1}\right)\right]} \\
\leq & \left|\alpha_{n-1}-\alpha_{n}\right|\left[\gamma\left\|P_{C}\left(I-\gamma \nabla f_{\alpha_{n}}\right) x_{n-1}\right\|+5 \gamma\left\|x_{n-1}\right\|\right] \\
\leq & M 3\left|\alpha_{n-1}-\alpha_{n}\right| .
\end{aligned}
$$

So,

$$
\left\|x_{n+1}-x_{n}\right\| \leq\left[1-(1-\rho) \theta_{n}\right]\left\|x_{n}-x_{n-1}\right\|+M_{2}\left|\theta_{n}-\theta_{n-1}\right|+M_{3}\left|\alpha_{n-1}-\alpha_{n}\right|,
$$

by Lemma 2.5 ,

$$
\left\|x_{n+1}-x_{n}\right\| \rightarrow 0
$$

$\left(3^{\circ}\right)$ Next we show that $\left\|x_{n}-T_{n} x_{n}\right\| \rightarrow 0$.

Indeed, it follows that

$$
\begin{aligned}
\left\|x_{n}-T_{n} x_{n}\right\| & \leq\left\|x_{n}-x_{n+1}\right\|+\left\|x_{n+1}-T_{n} x_{n}\right\| \\
& =\left\|x_{n}-x_{n+1}\right\|+\left\|P_{c}\left[\theta_{n} h\left(x_{n}\right)+\left(1-\theta_{n}\right) T_{n}\left(x_{n}\right)\right]-P_{C} T_{n}\left(x_{n}\right)\right\| \\
& \leq\left\|x_{n}-x_{n+1}\right\|+\theta_{n}\left\|h\left(x_{n}\right)-T_{n} x_{n}\right\| \rightarrow 0 .
\end{aligned}
$$

Now we show that

$$
\limsup _{n \rightarrow \infty}\left\langle h\left(x^{*}\right)-x^{*}, x_{n}-x^{*}\right\rangle \leq 0 .
$$

Let $x_{n_{k}} \rightarrow \tilde{x}$, observe that

$$
\begin{aligned}
\left\|P_{C}\left(I-\gamma \nabla f_{\alpha_{n}}\right) x_{n}-x_{n}\right\| & =\left\|\lambda_{n} I+\left(1-\lambda_{n}\right) T_{n} x_{n}-x_{n}\right\| \\
& =\left(1-\lambda_{n}\right)\left\|T_{n} x_{n}-x_{n}\right\| \\
& \leq\left\|T_{n} x_{n}-x_{n}\right\|,
\end{aligned}
$$

hence we have

$$
\begin{aligned}
& \left\|P_{C}(I-\gamma \nabla f) x_{n}-x_{n}\right\| \\
& \quad \leq\left\|P_{C}(I-\gamma \nabla f) x_{n}-P_{C}\left(I-\gamma \nabla f_{\alpha_{n}}\right) x_{n}\right\|+\left\|P_{C}\left(I-\gamma \nabla f_{\alpha_{n}}\right) x_{n}-x_{n}\right\| \\
& \quad \leq \gamma \alpha_{n}\left\|x_{n}\right\|+\left\|T_{n} x_{n}-x_{n}\right\| \rightarrow 0 .
\end{aligned}
$$


So

$$
\lim _{n \rightarrow \infty}\left\|P_{C}(I-\gamma \nabla f) x_{n}-x_{n}\right\|=0,
$$

by Lemma 2.4 and $\lim _{n \rightarrow \infty}\left\|P_{C}(I-\gamma \nabla f) x_{n}-x_{n}\right\|=0$, then

$$
\tilde{x}=P_{C}(I-\gamma \nabla f)(\tilde{x}) .
$$

This shows that

$$
\limsup _{n \rightarrow \infty}\left\langle h\left(x^{*}\right)-x^{*}, x_{n}-x^{*}\right\rangle \leq 0 .
$$

It follows that

$$
\begin{aligned}
&\left\|x_{n+1}-x^{*}\right\|^{2} \\
&=\left\|P_{c}\left[\theta_{n} h\left(x_{n}\right)+\left(1-\theta_{n}\right) T_{n}\left(x_{n}\right)\right]-P_{c} x^{*}\right\|^{2} \\
& \leq\left\|\theta_{n}\left(h\left(x_{n}\right)-x^{*}\right)+\left(1-\theta_{n}\right)\left(T_{n} x_{n}-T x^{*}\right)\right\|^{2} \\
&=\left\|\theta_{n}\left(h\left(x_{n}\right)-h\left(x^{*}\right)\right)+\left(1-\theta_{n}\right)\left(T_{n} x_{n}-T x^{*}\right)+\theta_{n}\left(h\left(x^{*}\right)-x^{*}\right)\right\|^{2} \\
& \leq\left\|\theta_{n}\left(h\left(x_{n}\right)-h\left(x^{*}\right)\right)+\left(1-\theta_{n}\right)\left(T_{n} x_{n}-T x^{*}\right)\right\|^{2}+2 \theta_{n}\left\langle h\left(x^{*}\right)-x^{*}, x_{n+1}-x^{*}\right\rangle \\
& \leq \theta_{n}\left\|h\left(x_{n}\right)-h\left(x^{*}\right)\right\|^{2}+\left(1-\theta_{n}\right)\left\|\left(T_{n} x_{n}-x^{*}\right)\right\|^{2}+2 \theta_{n}\left\langle h\left(x^{*}\right)-x^{*}, x_{n+1}-x^{*}\right\rangle \\
& \leq \theta_{n} \rho^{2}\left\|x_{n}-x^{*}\right\|^{2}+\left(1-\theta_{n}\right)\left\|\left(T_{n} x_{n}-T_{n} x^{*}+T_{n} x^{*}-T x^{*}\right)\right\|^{2} \\
&+2 \theta_{n}\left\langle h\left(x^{*}\right)-x^{*}, x_{n+1}-x^{*}\right\rangle \\
& \leq \theta_{n} \rho^{2}\left\|x_{n}-x^{*}\right\|^{2}+\left(1-\theta_{n}\right)\left[\left\|x_{n}-x^{*}\right\|+\alpha_{n} M\left(x^{*}\right)\right]^{2}+2 \theta_{n}\left\langle h\left(x^{*}\right)-x^{*}, x_{n+1}-x^{*}\right\rangle \\
& \leq {\left[1-(1-\rho) \theta_{n}\right]\left\|x_{n}-x^{*}\right\|^{2}+\left(1-\theta_{n}\right)\left[2 \alpha_{n} M\left(x^{*}\right)\left\|x_{n}-x^{*}\right\|+\alpha_{n}^{2}\left(M\left(x^{*}\right)\right)^{2}\right] } \\
&+2 \theta_{n}\left\langle h\left(x^{*}\right)-x^{*}, x_{n+1}-x^{*}\right\rangle .
\end{aligned}
$$

Hence,

$$
\begin{aligned}
& \left\|x_{n+1}-x^{*}\right\|^{2}=\left(1-\beta_{n}\right)\left\|x_{n}-x^{*}\right\|^{2}+\beta_{n} \delta_{n}, \\
& \beta_{n}=(1-\rho) \theta_{n}, \\
& \delta_{n}=\frac{1}{1-\rho}\left[\frac{\alpha_{n}}{\theta_{n}}\left(1-\theta_{n}\right) 2 M\left(x^{*}\right)\left\|x_{n}-x^{*}\right\|+\left(1-\theta_{n}\right)\left(M\left(x^{*}\right)\right)^{2} \frac{\alpha_{n}^{2}}{\theta_{n}}\right. \\
& \left.\quad+2\left\langle h\left(x^{*}\right)-x^{*}, x_{n+1}-x^{*}\right\rangle\right],
\end{aligned}
$$

by Lemma 2.5 and $\lim _{n \rightarrow \infty} \beta_{n}=0, \sum_{n=0}^{\infty} \beta_{n}=\infty$, $\lim _{\sup _{n \rightarrow \infty}} \delta_{n} \leq 0$, we have $x_{n} \rightarrow x^{*}$.

\section{Application of the iterative method}

Next, we give an application of Theorem 3.2 to the split feasibility problem (say SFP, for short) which was introduced by Censor and Elfving [15].

Find $x \in C$ such that $A x \in Q$, 
where $C$ and $Q$ are nonempty closed convex subsets of Hilbert spaces $H_{1}$ and $H_{2}$, respectively. $A: H_{1} \rightarrow H_{2}$ is a bounded linear operator.

It is clear that $x^{*}$ is a solution of split feasibility problem (18) if and only if $x^{*} \in C$ and $A x^{*}-P_{Q} A x^{*}=0$.

We define the proximity function $f$ by

$$
f(x)=\frac{1}{2}\left\|A x-P_{Q} A x\right\|^{2},
$$

and consider the convex optimization problem

$$
\min _{x \in C} f(x)=\min _{x \in C} \frac{1}{2}\left\|A x-P_{Q} A x\right\|^{2} .
$$

Then $x^{*}$ solves split feasibility problem (18) if and only if $x^{*}$ solves minimization (19) with minimizer equal to 0 . Byrne [16] introduced the so-called $C Q$ algorithm to solve the (SFP)

$$
x_{n+1}=P_{C}\left(I-\mu A^{*}\left(I-P_{Q}\right) A\right) x_{n}, \quad n \geq 0,
$$

where $0<\mu<\frac{2}{\left\|A^{*} A\right\|}=\frac{2}{\|A\|^{2}}$.

He obtained that the sequence $\left\{x_{n}\right\}$ generated by (20) converges weakly to a solution of the (SFP).

Now we consider the regularization technique. Let

$$
f_{\alpha}(x)=\frac{1}{2}\left\|A x-P_{Q} A x\right\|^{2}+\frac{\alpha}{2}\|x\|^{2},
$$

then we establish the iterative scheme as follows:

$$
x_{n+1}=P_{C}\left[\theta_{n} h\left(x_{n}\right)+\left(1-\theta_{n}\right) T_{n} x_{n}\right], \quad n \geq 0,
$$

where $h: C \rightarrow H_{1}$ is a contraction with $0<\rho<1$,

$$
\begin{aligned}
& P_{C}\left[I-\mu\left(A^{*}\left(I-P_{Q}\right) A+\alpha_{n} I\right)\right]=\lambda_{n} I+\left(1-\lambda_{n}\right) T_{n}, \\
& \lambda_{n}=\frac{2-\mu\left(\|A\|^{2}+\alpha_{n}\right)}{4} .
\end{aligned}
$$

Applying Theorem 3.2, we obtain the following result.

Theorem 4.1 Assume that the split problem is consistent, let the sequence $\left\{x_{n}\right\}$ be generated by

$$
x_{n+1}=P_{C}\left[\theta_{n} h\left(x_{n}\right)+\left(1-\theta_{n}\right) T_{n} x_{n}\right], \quad n \geq 0,
$$

where

$$
\begin{aligned}
& P_{C}\left[I-\mu\left(A^{*}\left(I-P_{Q}\right) A+\alpha_{n} I\right)\right]=\lambda_{n} I+\left(1-\lambda_{n}\right) T_{n}, \\
& \lambda_{n}=\frac{2-\mu\left(\|A\|^{2}+\alpha_{n}\right)}{4},
\end{aligned}
$$

and the sequence $\left\{\theta_{n}\right\} \subset(0,1),\left\{\alpha_{n}\right\}$ and $\left\{\theta_{n}\right\}$ satisfy the following conditions: 
(i) $\theta_{n} \rightarrow 0 ; 0<\mu<\frac{2}{\left\|A^{*} A\right\|}=\frac{2}{\|A\|^{2}}$;

(ii) $\sum_{n=0}^{\infty} \theta_{n}=\infty$;

(iii) $\sum_{n=0}^{\infty}\left|\theta_{n+1}-\theta_{n}\right|<\infty$;

(iv) $\sum_{n=0}^{\infty}\left|\alpha_{n+1}-\alpha_{n}\right|<\infty$;

(v) $\alpha_{n}=o\left(\theta_{n}\right)$.

Then the sequence $\left\{x_{n}\right\}$ converges strongly to the solution of split feasibility problem (18).

Proof By the definition of the proximity function $f$, we have

$$
\nabla f(x)=A^{*}\left(I-\operatorname{Proj}_{Q}\right) A x,
$$

and $\nabla f$ is $1 /\|A\|^{2}$-ism, i.e., since $\operatorname{Proj}_{Q}$ is $1 / 2$-averaged mapping, then $I-\operatorname{Proj}_{Q}$ is 1 -ism,

$$
\begin{aligned}
&\langle\nabla f(x)-\nabla f(y), x-y\rangle-1 /\|A\|^{2} \cdot\|\nabla f(x)-\nabla f(y)\|^{2} \\
&=\left\langle A^{*}\left(I-\operatorname{Proj}_{Q}\right) A x-A^{*}\left(I-\operatorname{Proj}_{Q}\right) A y, x-y\right\rangle \\
& \quad-1 /\|A\|^{2} \cdot\left\|A^{*}\left(I-\operatorname{Proj}_{Q}\right) A x-A^{*}\left(I-\operatorname{Proj}_{Q}\right) A y\right\|^{2} \\
&=\left\langle A^{*}\left[\left(I-\operatorname{Proj}_{Q}\right) A x-\left(I-\operatorname{Proj}_{Q}\right) A y\right], x-y\right\rangle \\
& \quad-1 /\|A\|^{2} \cdot\left\|A^{*}\left[\left(I-\operatorname{Proj}_{Q}\right) A x-\left(I-\operatorname{Proj}_{Q}\right) A y\right]\right\|^{2} \\
&=\left\langle\left(I-\operatorname{Proj}_{Q}\right) A x-\left(I-\operatorname{Proj}_{Q}\right) A y, A x-A y\right\rangle \\
& \quad-1 /\|A\|^{2} \cdot\left\|A^{*}\left[\left(I-\operatorname{Proj}_{Q}\right) A x-\left(I-\operatorname{Proj}_{Q}\right) A y\right]\right\|^{2} \\
& \geq\left\|\left(I-\operatorname{Proj}_{Q}\right) A x-\left(I-\operatorname{Proj}_{Q}\right) A y\right\|^{2} \\
&-\left\|\left(I-\operatorname{Proj}_{Q}\right) A x-\left(I-\operatorname{Proj}_{Q}\right) A y\right\|^{2} \\
&= 0 .
\end{aligned}
$$

Hence, $\langle\nabla f(x)-\nabla f(y), x-y\rangle \geq 1 /\|A\|^{2} \cdot\|\nabla f(x)-\nabla f(y)\|^{2}$.

Set $f_{\lambda_{n}}(x)=f(x)+\frac{\alpha}{2}\|x\|^{2}$; consequently,

$$
\begin{aligned}
\nabla f_{\alpha}(x) & =\nabla f(x)+\alpha I(x) \\
& =A^{*}\left(I-\operatorname{Proj}_{Q}\right) A x+\alpha x .
\end{aligned}
$$

Let $\gamma=\mu, L=\|A\|^{2}$, then the iterative scheme is equivalent to

$$
x_{n+1}=P_{C}\left[\theta_{n} h\left(x_{n}\right)+\left(1-\theta_{n}\right) T_{n} x_{n}\right], \quad n \geq 0,
$$

where $0<\gamma<\frac{2}{L}, P_{c}\left[I-\gamma \nabla f_{\alpha_{n}}\right]=\lambda_{n} I+\left(1-\lambda_{n}\right) T_{n}$ and $\lambda_{n}=\frac{2-\gamma\left(L+\alpha_{n}\right)}{4}$.

Due to Theorem 3.2, we have the conclusion immediately. 


\section{Acknowledgements}

The author thanks the referees for their helpful comments, which notably improved the presentation of this manuscript. This work was supported by the Fundamental Research Funds for the Central Universities (the Special Fund of Science in Civil Aviation University of China: No. 3122013K004).

Received: 2 January 2013 Accepted: 10 October 2013 Published: 22 Nov 2013

\section{References}

1. Levitin, ES, Polyak, BT: Constrained minimization methods. Zh. Vychisl. Mat. Mat. Fiz. 6, 787-823 (1966)

2. Calamai, PH, More, JJ: Projected gradient methods for linearly constrained problem. Math. Program. 39, 93-116 (1987)

3. Polyak, BT: Introduction to Optimization. Optimization Software, New York (1987)

4. Su, M, Xu, HK: Remarks on the gradient-projection algorithm. J. Nonlinear Anal. Optim. 1, 35-43 (2010)

5. Moudafi, A: Viscosity approximation methods for fixed-points problems. J. Math. Anal. Appl. 241, 46-55 (2000)

6. Xu, HK: Viscosity approximation methods for nonexpansive mappings. J. Math. Anal. Appl. 298, 279-291 (2004)

7. Ceng, LC, Ansari, QH, Yao, JC: Some iterative methods for finding fixed points and for solving constrained convex minimization problems. Nonlinear Anal. TMA 74, 5286-5302 (2011)

8. Marino, G, Xu, HK: Convergence of generalized proximal point algorithm. Commun. Pure Appl. Anal. 3, 791-808 (2004)

9. Marino, G, Xu, HK: A general iterative method for nonexpansive mappings in Hilbert spaces. J. Math. Anal. Appl. 318, 43-52 (2006)

10. Tian, M: A general iterative algorithm for nonexpansive mappings in Hilbert spaces. Nonlinear Anal. TMA 73, 689-694 (2010)

11. Yao, Y, Liou, YC, Chen, CP: Algorithms construction for nonexpansive mappings and inverse-strongly monotone mapping. Taiwan. J. Math. 15, 1979-1998 (2011)

12. Yao, Y, Chen, R, Liou, Y-C: A unified implicit algorithm for solving the triple-hierarchical constrained optimization problem. Math. Comput. Model. 55, 1506-1515 (2012)

13. Yao, Y, Liou, Y-C, Kang, SM: Two-step projection methods for a system of variational inequality problems in Banach spaces. J. Glob. Optim. (2013). doi:10.1007/s10898-011-9804-0

14. Wiyada, K, Praairat, J, Poom, K: Generalized systems of variational inequalities and projection methods for inverse-strongly monotone mapping. Discrete Dyn. Nat. Soc. (2011). doi:10.1155/2011/976505

15. Censor, Y, Elfving, T: A multiprojection algorithm using Bregman projections in a product space. Numer. Algorithms 8 , 221-239 (1994)

16. Byrne, C: A unified treatment of some iterative algorithms in signal processing and image reconstruction. Inverse Probl. 20, 103-120 (2004)

17. Censor, Y, Elfving, T, Kopf, N, Bortfeld, T: The multiple-sets split feasibility problem and its applications for inverse problem. Inverse Probl. 21, 2071-2084 (2005)

18. Censor, Y, Bortfeld, T, Martin, B, Trfimov, A: A unified approach for inversion problems in intensity-modulated radiation therapy. Phys. Med. Biol. 51, 2353-2365 (2006)

19. Xu, HK: A variable Krasnosel'skii-Mann algorithm and the multiple-set split feasibility problem. Inverse Probl. 22 2021-2034 (2006)

20. $\mathrm{Xu}, \mathrm{HK}$ : Iterative methods for the split feasibility problem in infinite-dimensional Hilbert space. Inverse Probl. 26 $105018(2010)$

21. Lopez, G, Martin, V, Xu, HK: Perturbation techniques for nonexpansive mapping with applications. Nonlinear Anal., Real World Appl. 10, 2369-2383 (2009)

22. Lopez, G, Martin, V, Xu, HK: Iterative algorithms for the multiple-sets split feasibility problem. In: Censor, Y, Jiang, M, Wang, G (eds.) Biomedical Mathematics: Promising Directions in Imaging, Therapy Planning and Inverse Problems, pp. 243-279. Medical Physica Publishing, Madison (2009)

23. Xu, HK: Averaged mapping and the gradient-projection algorithm. J. Optim. Theory Appl. 150, 360-378 (2011). doi:10.1007/s10957-011-9837-z

24. Xu, HK: A regularization method for the proximal point algorithm. J. Glob. Optim. 36, 115-125 (2006)

25. Cho, YJ, Petrot, N: Regularization and iterative method for general variational inequality problem in Hilbert spaces. J. Inequal. Appl. 2011, 21 (2011)

26. Combettes, PL: Solving monotone inclusions via composition of nonexpansive averaged operators. Optimization 53(5-6), 475-504 (2004)

27. Geobel, K, Kirk, WA: Topics on Metric Fixed-Point Theory. Cambridge Studies in Advanced Mathematics, vol. 28. Cambridge University Press, Cambridge (1990) 